15.1

\title{
Определение времени продольной релаксации текущей жидкости ядерно-магнитным спектрометром дифференциального типа
}

\author{
() В.В. Давыдов ${ }^{1,2}$, Н.С. Мязин ${ }^{1}$, В.И. Дудкин ${ }^{3}$, Р.В. Давыдов ${ }^{1}$ \\ ${ }^{1}$ Санкт-Петербургский политехнический университет Петра Великого, Санкт-Петербург, Россия \\ ${ }^{2}$ Всероссийский научно-исследовательский институт фитопатологии, Большие Вяземы, Московская обл., Россия \\ ${ }^{3}$ Санкт-Петербургский государственный университет телекоммуникаций им. проф. М.А. Бонч-Бруевича, Санкт-Петербург, \\ Россия \\ E-mail: Davydov_vadim66@mail.ru
}

Поступило в Редакцию 19 сентября 2019 г.

В окончательной редакции 17 августа 2020 г.

Принято к публикации 17 августа 2020 г.

\begin{abstract}
Обоснована необходимость одновременного измерения значений времен продольной $T_{1}$ и поперечной $T_{2}$ релаксации для контроля состояния текущей жидкости при проведении исследования в диапазоне изменения расхода $q$ минимум в два порядка. Разработан новый метод измерения $T_{1}$. Для его реализации предложена конструкция ядерно-магнитного спектрометра дифференциального типа, позволяющая проводить измерения $T_{1}$ во всем диапазоне измерения расхода $q$. Представлены результаты экспериментальных исследований.
\end{abstract}

Ключевые слова: ядерно-магнитный спектрометр, время продольной релаксации $T_{1}$, намагниченность, текущая жидкость, состояние среды.

DOI: 10.21883/PJTF.2020.22.50309.18043

Разработка быстрых и надежных методов контроля состояния текущей среды в широком диапазоне изменения ее расхода $q$ является одной из актуальных задач технической физики [1-4]. Контролировать состояние текущей среды необходимо как при проведении экспериментов, так и при автоматизации промышленного производства различных жидких сред, биологических растворов и т.д. $[3,5,6]$. Результаты исследований показали, что наиболее эффективным и целесообразным является контроль состояния текущей среды по измеренным значениям времен продольной $T_{1}$ и поперечной $T_{2}$ релаксации с использованием явления ядерного магнитного резонанса (ЯМР) [7-11].

По причине того, что устройства контроля состояния текущей среды должны эффективно работать в диапазоне изменения $q$ минимум два порядка, при измерении $T_{1}$ и $T_{2}$ возникает ряд проблем, основная из которых связана с ограничениями, возникающими при использовании ранее разработанных методик для измерения значений $T_{1}$ в жидкой среде [7,9-13], так как при проведении экспериментов или контроле технологического процесса достаточно сложно обеспечить проведение измерений $T_{1}$ при оптимальном значении расхода $q_{\text {opt }}[9,10]$ с изменением значений $q$ в пределах двух порядков и более. Кроме того, проведенные нами эксперименты позволили установить, что для обеспечения максимального отношения сигнал/шум и разрешения регистрируемого сигнала ЯМР в форме „виглей“ с использованием модуляционной методики должны выполняться следующие соотношения:

$$
\begin{gathered}
t_{r}>2 / f_{m}, \\
\gamma B_{m}>10 \Delta f_{n m r}, \\
1 / f_{m}>6 T_{2},
\end{gathered}
$$

где $B_{m}-$ амплитуда поля модуляции, $f_{m}-$ частота поля модуляции, $\Delta f_{n m r}$ - естественная ширина линии.

Выполнение приведенных выше соотношений создает (особенно при быстрых расходах жидкости) сложности с изменением значения $f_{m}$ на порядок для измерения $T_{1}$ методом изменения величины намагниченности $M$ жидкой среды, который успешно применяется при экспрессконтроле [11,12].

Метод, изложенный в работе [9], использующий дополнительный коэффициент в соотношении для определения $q_{\text {opt }}$, предложенном в [10], чтобы исключить „Привязку“ измерения $T_{1}$ к значению $q_{\text {opt }}$, показал низкую точность, так как значение вводимого коэффициента, который получается из уравнений Блоха [14], как показали эксперименты, зависит от изменения расхода жидкости $q$. В работе [9] авторы предлагают считать его постоянным в некотором диапазоне изменения $q$, что противоречит теории ЯМР и экспериментальным данным, особенно при больших значениях $q$.

Поэтому целью настоящей работы является разработка нового метода, который позволяет проводить измерения $T_{1}$ в текущей жидкости с погрешностью менее $1 \%$ в диапазоне изменения $q$ минимум в два порядка.

В конструкциях ЯМР-спектрометров и релаксометров с текущей средой сигнал ЯМР регистрируется с использованием модуляционной методики автодинным 


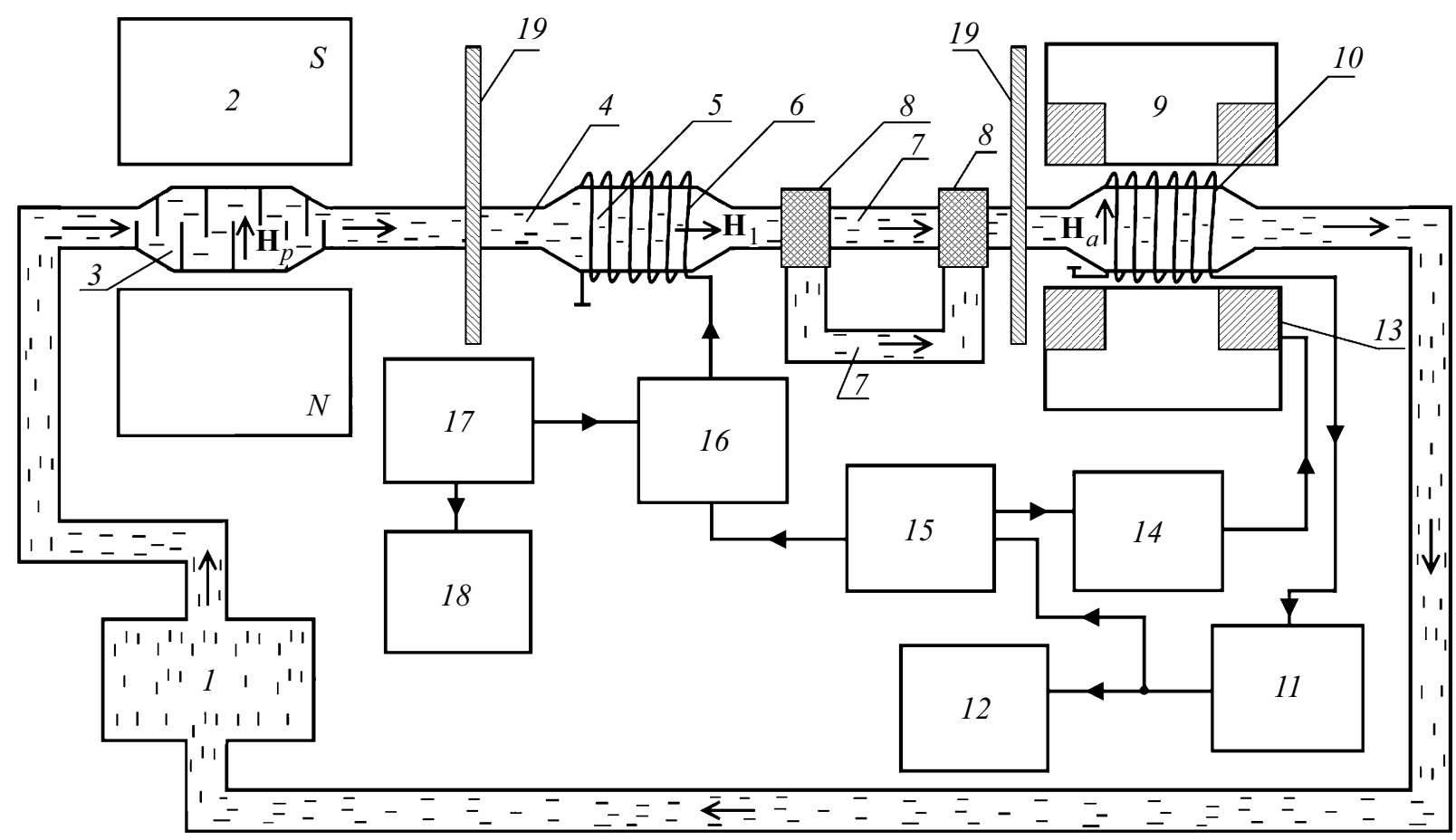

Рис. 1. Структурная схема ядерно-магнитного спектрометра. 1 - насос, 2 - магнит-поляризатор, 3 - сосуд-поляризатор, 4 - соединительный участок трубопровода, 5 - объем катушки нутации, 6 - катушка нутации, 7 - соединительный участок трубопровода, подключенный через переключатели потока, $8-$ переключатели потока, $9-$ магнит-анализатор, $10-$ катушка регистрации сигнала ЯМР, 11 - схема регистрации, 12 - осциллограф, 13 - катушки модуляции, 14 - генератор модуляции, 15 - схема обработки и управления, 16 - электронные ключи, 17 - генератор нутации, 18 - частотомер, 19 - магнитные экраны.

детектором [4,9-11,15]. С учетом особенностей работы автодинного детектора $[10,11,15]$ форма линии $G(t)$ регистрируемого сигнала ЯМР описывается следующим соотношением

$$
G(t)=F\left(\frac{A}{A+B} v^{2}(t)+\frac{B}{A+B} u^{2}(t)\right)^{1 / 2},
$$

где $v(t), u(t)$ - сигналы поглощения и дисперсии, $A$ и $B$ - коэффициенты, определяющие вклад в регистрируемый сигнал ЯМР от сигналов поглощения и дисперсии, а $F-$ коэффициент, учитывающий изменения фазы.

Сигналы поглощения и дисперсии для (1) получаются из решения уравнений Блоха, записанных во вращающейся системе координат [10,14]:

$$
\begin{gathered}
d u(t) / d t+u(t) / T_{2}+\gamma H_{m} \sin \left(\omega_{m} t\right) v(t)=0, \\
d v(t) / d t+v(t) / T_{2}-\gamma H_{m} \sin \left(\omega_{m} t\right) u(t)+\gamma H_{1} M_{z}(t)=0, \\
d M_{z}(t) / d t+M_{z}(t) / T_{1}-M / T_{1}-\gamma H_{1} v(t)=0,
\end{gathered}
$$

где $\Delta \omega=\omega_{0}-\omega_{n m r}-$ расстройка частоты регистрации сигнала ЯМР $\omega_{n m r}$ от частоты прецессии намагниченности ядер $M$ в поле $H_{a}\left(\omega_{0}=\gamma H_{a}, M=\chi_{0} H_{a}\right), \chi_{0}-$ статическая ядерная магнитная восприимчивость, $T_{1}$ и $T_{2}-$ времена продольной и поперечной релаксации жидкой среды, $t$ - время.
Система уравнений (2) решается относительно компонент $v(t), u(t)$ и $M_{z}(t)$ с учетом начальных условий $M_{z}(0)=M_{m}, v(0)=0, u(0)=0$, где $M_{m}$ - значение намагниченности жидкости на входе катушки регистрации ЯМР-спектрометра. Проведенные нами эксперименты показали, что значение амплитуды регистрируемого сигнала ЯМР зависит от изменения величины $M_{m}$. На величину намагниченности текущей жидкости в момент ее протекания между устройствами для намагничивания и регистрации сигнала ЯМР действуют релаксационные и другие процессы. Поэтому в задачу работы входила также разработка конструкции ЯМР спектрометра, в которой действие других процессов на изменение величины $M_{m}$ было бы несущественно.

На рис. 1 представлена структурная схема экспериментальной установки ЯМР-спектрометра дифференциального типа для реализации нового метода измерения $T_{1}$. Текущая жидкость с начальной намагниченностью $M_{0}$ поступает в сосуд-поляризатор 3 с объемом $V_{p}$, размещенный в магнитном поле $H_{p}$. На выходе из магнита-поляризатора 2 жидкость приобретает намагниченность $M_{1}$ :

$$
M_{1}=\chi_{0} H_{p}\left[1-\exp \left(-V_{p} /\left(q T_{1}\right)\right)\right]+M_{0} \exp \left(-V_{p} /\left(q T_{1}\right)\right) .
$$

Для намагничивания жидкости используют поля с индукцией $B_{p}>1$ T. Начальная намагниченность текущей 

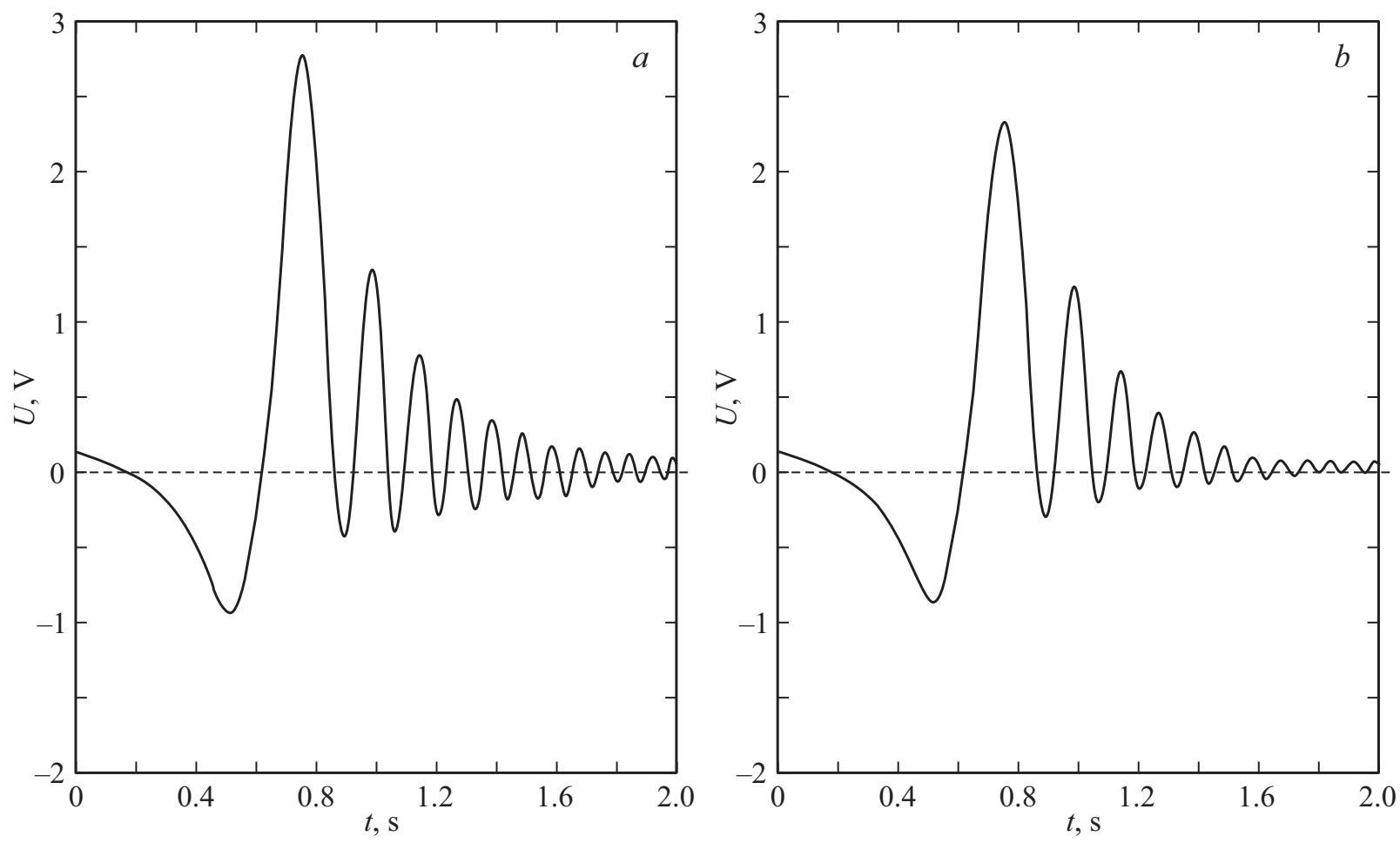

Рис. 2. Формы линий сигналов ЯМР на выходе схемы регистрации 11 от водопроводной воды при $f_{m}=50 \mathrm{~Hz}$ для различных объемов соединительного участка трубопровода $7: V_{c 1}=146 \mathrm{ml}(a)$ и $V_{c 2}=204 \mathrm{ml}(b)$.

среды $M_{0}$ формируется в поле Земли $\left(B_{E} \approx 0.5 \cdot 10^{-4} \mathrm{~T}\right)$. Поэтому вторым слагаемым в (3) можно пренебречь по сравнению с первым:

$$
M_{1}=\chi_{0} H_{p}\left[1-\exp \left(-V_{p} /\left(q T_{1}\right)\right)\right]
$$

В случае установки магнитных экранов изменение магнитного поля $H_{t}$ в пределах двух соединительных участков трубопровода 4 и 7 и катушки нутации 6 несущественно по сравнению с неоднородностью магнитного поля поляризатора 2. Поэтому при рассмотрении можно взять ее среднюю величину в зоне размещения катушки нутации 6 . В этом случае значение $M_{m}$ можно представить следующим выражением:

$$
\begin{aligned}
M_{m}= & \chi_{0} H_{t}\left[1-\exp \left(-\left(V_{m}+V_{n}+V_{c}\right) /\left(q T_{1}\right)\right)\right] \\
& +M_{1} \exp \left[-\left(V_{m}+V_{n}+V_{c}\right) /\left(q T_{1}\right)\right],
\end{aligned}
$$

где $V_{m}$ - объем соединительного участка трубопровода 4 (рис. 1), $V_{n}$ - объем катушки нутации 5 (рис. 1), $V_{c}$ - объем соединительного участка трубопровода 7 (рис. 1).

Проведенные нами эксперименты показали, что при $B_{a} \approx 0.3 \mathrm{~T}$ и $B_{p}>1 \mathrm{~T}$ значение $B_{t}<0.0002 \mathrm{~T}$. Кроме того, текущая жидкость в объеме $V_{m}+V_{n}+V_{c}$ находится в течение времени, которое много меньше $T_{1}$, что не позволяет ей полностью намагнититься в отличие от случая ее протекания через сосуд-поляризатор 3 [1,4,10,14]. Поэтому значения $M_{1}$ и намагниченности $M_{t}=\chi_{0} H_{t}$, которая образуется на этом участке трубопровода, будут различаться между собой более чем на четыре порядка. В этом случае первым слагаемым в (5) можно пренебречь по сравнению со вторым, и выражение для определения $M_{m}$ приобретает следующий вид:

$$
\begin{aligned}
M_{m}= & \chi_{0} H_{p}\left[1-\exp \left(-V_{p} /\left(q T_{1}\right)\right)\right] \exp \left[-\left(V_{m}+V_{n}\right) /\left(q T_{1}\right)\right] \\
& \times \exp \left[-\left(V_{c}\right) /\left(q T_{1}\right)\right] .
\end{aligned}
$$

Если использовать два участка соединительного трубопровода с разными объемами $V_{c}$, то регистрируемые сигналы ЯМР от текущей жидкости будут различаться по амплитуде, так как, согласно (6), $M_{m}$ изменяется. На рис. 2 в качестве примера представлены регистрируемые сигналы ЯМР от водопроводной воды при $T=293.2 \mathrm{~K}$ для двух объемов $V_{c}$ соединительных участков трубопровода. Анализ полученных форм линий сигналов ЯМР показывает, что в случае увеличения $V_{c}$ амплитуда регистрируемого сигнала ЯМР уменьшается, что соответствует (1) с учетом (2) и (6). Для подтверждения полученных данных были исследованы формы линий сигналов ЯМР с инверсией намагниченности $[4,7,10]$, которая создается в катушке нутации 6. На рис. 3 в качестве примера представлены регистрируемые сигналы ЯМР от водопроводной воды с инверсией намагниченности при двух значениях $V_{c}$. В результате исследований было установлено, что характер изменения амплитуды регистрируемого сигнала ЯМР при изменении значения $V_{c}$ для сигнала ЯМР с 

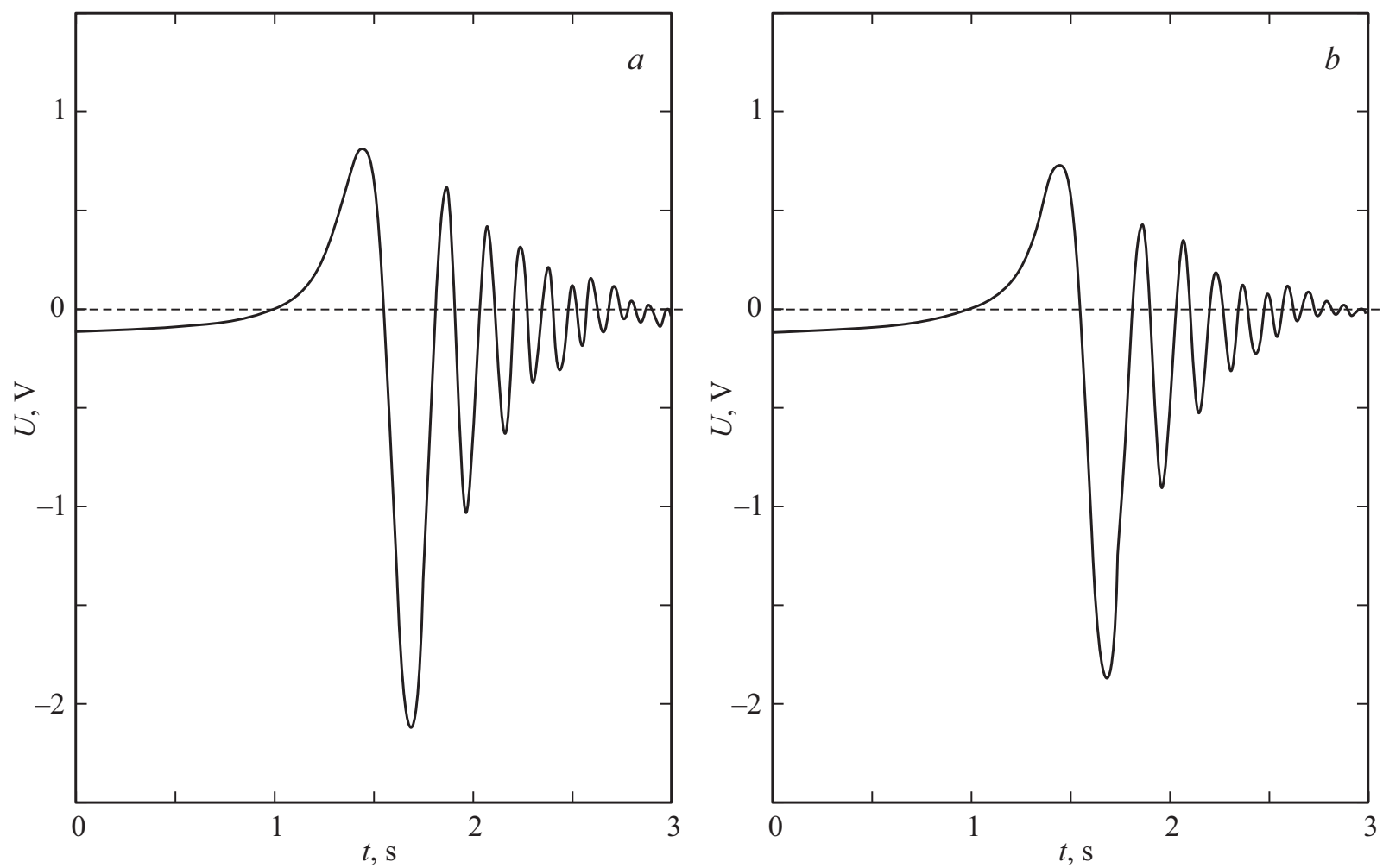

Рис. 3. Формы линий сигналов ЯМР с инверсией намагниченности на выходе схемы регистрации 11 от водопроводной воды при $f_{m}=50 \mathrm{~Hz}$ для различных объемов соединительного участка трубопровода $7: V_{c 1}=146 \mathrm{ml}(a)$ и $V_{c 2}=204 \mathrm{ml}(b)$.

инверсией намагниченности такой же, как без нее. Это означает, что на всем участке протекания намагниченной жидкости от сосуда-поляризатора до сосуда-анализатора выполняется условие адиабатической теоремы [16,17]. Величина намагниченности изменяется только под действием релаксационных процессов, остальные факторы оказывают несущественное влияние. Поэтому если провести измерения значений двух амплитуд ( $U_{1}$ и $\left.U_{2}\right)$ сигналов ЯМР при различных значениях $V_{c}$, то для определения значения $T_{1}$ можно использовать следующее соотношение:

$$
\frac{U_{1}}{U_{2}}=\frac{M_{m 1}}{M_{m 2}}=\frac{\exp \left(-\frac{V_{c 1}}{q T_{1}}\right)}{\exp \left(-\frac{V_{c 2}}{q T_{1}}\right)}
$$

Поскольку условия протекания жидкой среды по участку трубопровода и в катушке нутации для двух случаев подключения объемов $V_{c 1}$ и $V_{c 2}$ идентичны, соотношение (7) можно преобразовать к следующему виду:

$$
\frac{U_{1}}{U_{2}}=\frac{\exp \left(-\frac{V_{c 1}}{q T_{1}}\right)}{\exp \left(-\frac{V_{c 2}}{q T_{1}}\right)} .
$$

Выражение для определения $T_{1}$ по измеренным значениям $U_{1}, U_{2}$ и $q$ после преобразования приобретает вид

$$
T_{1}=\frac{V_{c 2}-V_{c 1}}{q \ln \left(\frac{U_{1}}{U_{2}}\right)} .
$$

Для водопроводной воды при $T=276.3 \mathrm{~K}$ с использованием разработанного нами нового метода (соотношение (9)) [9,10,15] было измерено значение $T_{1}=1.44 \pm 0.02 \mathrm{~s}$. Цикл измерения времен релаксаций повторялся 10 раз для усреднения данных и оценки погрешности измерения в соответствии со стандартными методиками. Аналогичная проба воды при $T=276.3 \mathrm{~K}$ была исследована на стационарном ЯМР-релаксометре Minispec mq 20 (фирма BRUKER), при этом измеренное значение $T_{1}=1.434 \pm 0.003 \mathrm{~s}$. Полученные значения $T_{1}$ совпали в пределах погрешности измерений.

Анализ экспериментальных результатов показал, что разработанный нами метод позволяет проводить измерения $T_{1}$ в реальном времени одновременно с измерениями $T_{2}$ для расходов жидкости $q$, при которых отношение сигнал/шум > 3. Это существенно расширяет возможности использования ЯМР-спектрометров для проведения научных исследований и решения других задач.

\section{Финансирование работы}

Работа поддержана Санкт-Петербургским политехническим университетом Петра Великого в рамках программы ,5-100-2020“.

\section{Конфликт интересов}

Авторы заявляют, что у них нет конфликта интересов. 


\section{Список литературы}

[1] Дьяченко С.В., Кондрашкова И.С., Жерновой А.И. // ЖТФ. 2017. Т. 87. В. 10. С. 1596-1598. DOI: 10.21883/JTF.2017.10.45007.2213 [Пер. версия: https://doi.org/10.1134/S1063784217100097].

[2] Давыдов В.В., Дудкин В.И., Карсеев А.Ю. // Изв. вузов. Физика. 2015. Т. 58. № 2. С. 8-13. [Пер. версия: https://doi.org/10.1007/s11182-015-0475-z].

[3] Даев Ж.А. // Измерительная техника. 2016. № 3. С. 29-31.

[4] Давыдов В.В., Дудкин В.И., Карсеев А.Ю. // Письма в ЖТФ. 2014. Т. 40. В. 19. С. 96-103. [Пер. версия: https://doi.org/10.1134/S1063785014100058].

[5] Popovac M., Hanjalic K. // Flow Turbul. Combust. 2007. V. 78. N 2. P. 177-184.

[6] Карабегов М.А. // Измерительная техника. 2012. № 11. C. $50-55$.

[7] Kashaev R.S., Gazizov E.G. // J. Appl. Spectr. 2010. V. 77. N 3. P. 321-328.

[8] Алакшин Е.М., Кондратьева Е.И., Кузьмин В.В., Сафиуллин К.Р., Станиславовас А.А., Савинков А.В., Клочков А.В., Тагиров М.С. // Письма в ЖЭТФ. 2018. Т. 107. B. 2. C. $115-118$. DOI: $10.7868 / \mathrm{S} 0370274 \mathrm{X} 1802008 \mathrm{X}$

[9] Fatkhutdinova L.I., Mamonkina A.N., Ermak S.V., Semenov V.V. // J. Phys.: Conf. Ser. 2018. V. 1124. N 5. P. 051043.

[10] Жерновой А.И., Латышев Г.Д. Ядерный магнитный резонанс в проточной жидкости. М.: Атомиздат, 1964. 254 с.

[11] Давыдов В.В., Мязин Н.С., Величко Е.Н. // Письма в ЖТФ. 2017. Т. 43. В. 13. С. 34-42. [Пер. версия: https://doi.org/10.1134/S1063785017070033].

[12] Давыдов В.В., Мязин Н.С., Давыдова Т.И. // Дефектоскопия. 2017. № 7. С. 52-61.

[13] Семенов А.А., Карманенко С.Ф., Мелков А.А., Бобыль А.В., Сурис Р.А., Гальперин Ю.М., Иохансен Т.Х. // ЖТФ. 2001. Т. 71. В. 10. С. 13-19. [Пер. версия: 10.1134/1.1412054].

[14] Bloch F. // Phys. Rev. 1946. V. 70. N 7. P. 460-478.

[15] Давыдов В.В., Дудкин В.И., Карсеев А.Ю. // Письма в ЖТФ. 2015. Т. 41. В. 7. С. 103-110. [Пер. версия: doi.org/10.1134/S1063785015040057].

[16] Абрагам А. Ядерный магнетизм. М.: ИИЛ, 1967. 686 с.

[17] Леше А. Ядерная индукция. М.: ИИЛ, 1963. 684 с. 